\title{
Evaluating the Effect of Different Mixing Rules on Thermodynamic Properties in Different Mixtures
}

\author{
Fatemeh Fadaei Nobandegani ${ }^{1, \text { *, Abouzar Roeintan }}{ }^{2}$ \\ ${ }^{1}$ Department of Food Science and Technology, Fasa University, Fasa, Iran \\ ${ }^{2}$ Department of Chemistry, Emam Hossein University, Tehran, Iran
}

\section{Email address:}

Fatima_fadaei_84@yahoo.com (F. F. Nobandegani)

${ }^{*}$ Corresponding author

\section{To cite this article:}

Fatemeh Fadaei Nobandegani, Abouzar Roeintan. Evaluating the Effect of Different Mixing Rules on Thermodynamic Properties in Different Mixtures. American Journal of Mechanics and Applications. Vol. 8, No. 1, 2019, pp. 1-6. doi: 10.11648/j.ajma.20200801.11

Received: June 22, 2019; Accepted: July 16, 2019; Published: January 8, 2020

\begin{abstract}
The purpose of this paper is to evaluate the effect of five different mixing rules on the calculated thermodynamic properties including vapor pressure, density and excess property of several binary mixtures. These properties are calculated by ISM (Ihm-Song-Mason) and PHS (Perturb Hard Sphere) equations of state (EOS). Also we use two interaction parameters, $\mathrm{K}_{\mathrm{ij}}$ to improve the results. The results indicate that mixing rules can effect on predicted thermodynamic properties. The Fit (MADAR-1) mixing rule gives more acceptable values. when the mixture components are similar in size, different mixing rules often do not change the errors in calculated properties more than $2 \%-1 \%$. However, as the size similarity decreases, the effect of applied mixing rules becomes more important.
\end{abstract}

Keywords: Mixing Rule, Equation of State, Thermodynamic

\section{Introduction}

One of the most successful and widely used methods for relating the thermodynamic properties of the mixtures to those of the pure fluid is the so called-one-fluid theory. Its principle is to treat a mixture as a hypothetical pure substance whose characteristic parameters are obtained by interpolation between the parameters of the pure components. The interpolation formulas are usually referred to mixing rules [1]

Cubic equation of state (CEOS) is used to calculate thermodynamic properties of pure fluids and mixtures. CEOS can describe pure components reasonably well.

For application of Cubic equations of state (CEOS) to fluid mixtures, mixing rules are required to calculate the overall attractive energy and co-volume parameters (the ' $a$ ' and ' $b$ ' terms, respectively). The choice of mixing rules can have significant effects on the predictions from CEOS. Depending on whether the mixture consists of molecules of similar types and size, and are polar or non-polar, one mixing rules may give better predictions than another. [2]

Non ideal behavior of asymmetry molecules is largely determined by the interactions between pairs of molecules which can be described by intermolecular potentials. In a mixture, there will also be interactions between non-identical molecules. The unlike interactions are accounted for using mixing rules.

There are some mistakes in distinguishing between the terms "mixing" and "combining" rules. They are used interchangeably in the literature. However, Wei and Sadus [3] used the term "mixing rule" to indicate the expressions used to obtain parameters for the van der Waals one fluid theory, while the term "combining rule" was used for mathematical expressions used to obtain cross interaction values from pure component values.

The distance between particles $\mathrm{i}, \sigma_{\mathrm{ii}}$, scale the length of the interaction, and parameter $\varepsilon_{i i}$, scales the well depth of the potential. Interaction between dissimilar particles requires the generation of cross term parameters for $\sigma$ and $\varepsilon$.

To estimate the potential between non-identical molecules, the Lorentz-Berthelot mixing rules are frequently used to mix the parameters from the systems for the two pairs of identical molecules. This mixing rule uses an arithmetic rule for the collision diameter (Lorentz rule) and a geometric mean for the well depth (Berthelot rule). 


\section{Theoretical}

In this work, we investigated five different mixing rules: Lorentz-Berthelot, Halgren HHG, Tang-Toennies, Waldman-Hagler, and Fit (MADAR-2). The last rule were developed by Al-Matar and Rockstraw [4] based on a certain weighting matrix approach for devising mixing rules.

The Tang-Toennies rules belong to theoretically developed rules, while all other rules are empirical expressions developed to match experimental data with the prediction from the rules.

The mathematical forms of the rules implemented in this research are given below.

Lorentz-Berthelot. These rules are the most widely used. $[5,6]$ An arithmetic average is used for the collision diameter, while a geometric average is used for the well depth.

$$
\begin{gathered}
\delta \mathrm{ij}=1 / 2(\delta \mathrm{ii}+\delta \mathrm{jj}) \\
\xi \mathrm{ij}=(\xi \mathrm{ii} \xi \mathrm{jj})^{1 / 2}
\end{gathered}
$$

Tang-Toennies.[7] These rules are based on some quantum mechanical derivation and are the most successful equation among the group of theoretical rules. The problem with such

an approach is the incorporation of additional parameter sets (e.g. polarizability, ionization potentials, or dispersion force coefficients). This is contradictory to the purpose behind introducing mixing rules. Mixing rules are introduced to reduce the number of parameter sets in any force field, while theoretical rules introduce more parameters and some of them are not easily determined.

Waldman-Hagler. This rule utilizes a sixth power mean for collision diameter and a simplified form of the reduced sixth dispersion coefficient for the well depth. [8]

Fit (MADAR-2). A least-squares analysis was carried out for both $\sigma$ and $\varepsilon$ to determine the weight factors matrix [9] for the fit form. The data set of Kestin and co-workers [10-11] was used throughout. The power was fixed to the sixth power for theoretical considerations; however, it can be treated as an adjustable parameter. [12]:

We calculated the values of $\sigma$ from the following equation

$$
\sigma=0.809\left(V_{c}\right)^{1 / 3}
$$

Where $V_{c}$ is the critical volume. The values of $\varepsilon$ were evaluated using the following equation

$$
\frac{\varepsilon}{K}=\frac{T c}{1.2593}
$$

Also in recent years Delara Mohammad-Aghaie, investigated the Effect of Mixing Rules on Transport Properties of Gas Mixtures [13] and Richard Anthony McFarlane evaluated the new co-volume mixing rules for the Peng-Robinson Equation of State [14] also Yugong WU evaluated the mixing rules for dielectric constants of composite dielectrics by MC-FEM calculation on 3D cubic lattice [15].

The main aim of this work is to understand the effect of these five mixing rules on the accuracies of predicted thermodynamic properties, including density, excess molar volume, and vapor pressure of several binary mixtures. We have selected wide varieties of mixtures consisting of: noble gas mixture $\mathrm{Ar}+\mathrm{Kr}$, mixtures of Xenon + Ethane and Xenon+ Propane, refrigerant mixtures R32+R143a as representatives of slightly polar mixtures. Further mixtures

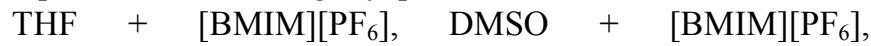
$[$ Emim $][$ Triflate $]+$ Propanol, and $[$ Emim $][$ Triflate $]+$ Water.

\section{Results and Discussion}

There have not been many studies to assess the effect of mixing rules on the accuracies of the predicted thermodynamic properties in the literature, so this study can provide a logical framework for selecting the most suitable mixing rules, based on an objective criterion. In our previous works [16] we employed ISM EOS and Purebred-HardSphere (PHS) EOS to calculated density and vapor pressure and excess molar volume of several mixtures, To calculate mixture thermodynamic properties with ISM EOS we need to know binary potential parameters $\sigma 12$ and $\varepsilon 12$, in terms of $\sigma$ and $\varepsilon$ of pure components. Mixing (combining) rules determine how the cross coefficients $\sigma 12$ and $\varepsilon 12$ are calculated. Maitlandet al. [17] provides a good review of the mixing rules literature prior to 1981 . In the present work more recent rules including those of Tang and Toennies [18], Halgren [7], Waldman and Hagler and also Al-Matar and Rockstraw [4] have been applied to check the effect of mixing rules on thermodynamic properties.

The common belief is that the mixing rules do not appreciably affect the outcome of a calculation, so there have not been many studies to assess the effect of mixing rules to the accuracy of thermos physical properties. The present work indicates that the results are slightly in favor of the Fit (MADAR-2) mixing rule, for thermodynamic properties of studied mixtures. On the other hand, this assessment shows the superiority of the Lorentz-Berthelot mixing rule in calculation of thermodynamic properties when the mixture components differ considerably in size.

\subsection{The ISM Equation of State}

The general frame of the Ihm-Song-Mason EOS read as:

$$
\frac{P}{\rho k T}=1+\frac{\left(B_{2}(T)-\alpha(T)\right) \rho}{1+0.22 \lambda b(T) \rho}+\frac{\alpha(T) \rho}{1-\lambda b(T) \rho}
$$

Where $P$ is the pressure, $\rho$ is the molar (number) density, $B_{2}(T)$ is the second virial coefficient, $\alpha(T)$ is the contribution of repulsive branch of pair potential function in the second virial coefficient. This is based on the recognition that the structure of a liquid is determined primarily by repulsive forces, so that fluids of hard bodies can serve as useful reference states. $b(\mathrm{~T})$ reflects the van der Waals co volume, $\mathrm{kT}$ is the thermal energy per one molecule. $\lambda$ is a free parameter which can be fixed with the help of volumetric properties of dense fluids. Any uncertainty in the parameters 
$\mathrm{B}_{2}, \alpha(\mathrm{T})$, and $\mathrm{b}(\mathrm{T})$ can be compensated for by selecting a proper value of $\lambda$ parameter.

$$
B_{2}(T)=2 \pi N_{A} \int_{0}^{\infty}\left(1-e^{-u(r) / k T}\right) r^{2} d r
$$

Where $\mathrm{N}_{\mathrm{A}}$ is Avogadro's number.

If a two-parameter potential energy function $u(r)$ can describe the nature of the interaction potential between particles of a fluid, the potential can be written in dimensionless form by:

$$
\frac{u}{\varepsilon}=F\left(\frac{r}{\sigma}\right)
$$

where $\varepsilon$ is a characteristic energy parameter and $\sigma$ is a characteristic size parameter, and $\mathrm{F}$ is a universal function of the reduced intermolecular separation. Upon substitution, Eq. (6) can be rewritten in dimensionless form:

$$
\frac{B_{2}(T)}{2 \pi N_{A} \sigma^{3}}=\int_{0}^{\infty}\left[1-\exp \left(\frac{-\varepsilon F(r / \sigma)}{k_{B} T}\right)\right]\left(\frac{r}{\sigma}\right)^{2} d\left(\frac{r}{\sigma}\right)
$$

It follows from the above equations that the second virial coefficients can be correlated by data reduction with energy and distance parameters as characteristic parameters. This equation also says that the reduced second virial coefficient is a generalized function of the reduced temperature. This function can be determined either by a direct correlation of experimental data for the second virial coefficient or by specification of the universal potential function $\mathrm{u} / \varepsilon$ and integration. In this work, we have taken $\varepsilon$ as the energy parameters and $\sigma$ as the size parameter in the potential energy relation.

The mixture version of the ISM EOS is as follows

$$
\frac{P}{\rho k T}=1+\rho \sum_{i} \sum_{j} x_{i} x_{j}\left(B_{i j}-\alpha_{i j}\right) F_{i j}+\rho \sum_{i} \sum_{j} x_{i} x_{j} G_{i j} \alpha_{i j}
$$

where $\alpha$ is a correction factor for the softness of the repulsive forces and $G$ is the average pair distribution function at contact for additive non-spherical hard-convex bodies. The summations run over all the components of mixtures and subscripts $i j$ refer to the $i-j$ interactions. The parameters $\mathrm{F}_{\mathrm{ij}}$ and $\mathrm{G}_{\mathrm{ij}}$ are defined as:

$$
\begin{gathered}
F_{\mathrm{ij}}=\frac{1}{1-\xi_{3}}-\left(\frac{b_{\mathrm{i}} b_{\mathrm{j}}}{b_{\mathrm{ij}}}\right)^{1 / 3} \frac{\rho \sum_{k} x_{k} b_{k}^{2 / 3}\left(0.22 \lambda_{k}+0.25\right)}{\left(1-\xi_{3}\right)\left(1+0.22 \rho \sum_{k} x_{k} b_{k} \lambda_{k}\right)} \\
G_{\mathrm{ij}}=\frac{1}{1-\xi_{3}}-\left(\frac{b_{\mathrm{i}} b_{\mathrm{j}}}{b_{\mathrm{ij}}}\right)^{1 / 3} \frac{\rho \sum_{k} x_{k} b_{k}^{2 / 3}\left(\lambda_{k}+0.25\right)}{\left(1-\xi_{3}\right)\left(1+\rho \sum_{k} x_{k} b_{k} \lambda_{k}\right)}
\end{gathered}
$$

With

$$
\xi_{3}=\frac{\rho}{4} \sum_{k} x_{k} b(T)_{k}
$$

The present method for calculating the second virial coefficient and the other two temperature-dependent parameters can be extended to mixtures by using a simple geometric mean for the non-bonded interaction energy parameter, $\varepsilon$ and a arithmetic mean for the effective hardsphere diameter:

$$
\begin{gathered}
(\varepsilon / k)_{\mathrm{ij}}=\sqrt{(\varepsilon / k)_{\mathrm{i}}(\varepsilon / k)_{\mathrm{j}}} \\
\sigma_{\mathrm{ij}}=\frac{1}{2}\left(\sigma_{\mathrm{i}}+\sigma_{\mathrm{j}}\right)
\end{gathered}
$$

The excess molar volume, $\mathrm{V}^{\mathrm{E}}$ of binary mixtures was calculated by taking the effects of additive hard-spheres.

And:

General frame of the PHS EOS has the following form (16, 17)

$$
\frac{P}{\rho k T}=\frac{1+\eta+\eta^{2}-\eta^{3}}{(1-\eta)^{3}}-\frac{a(T) \rho}{k T},
$$

where $P$ is the pressure, $\rho$ is the number (molar) density, $k T$ is the thermal energy per molecule and $\eta$ is packing fraction defined as:

$$
\eta=\frac{b(T) \rho}{4}
$$

Eq. (15) has two parts; 1) the Carnahan-Starling expression [19] taken as reference hard-sphere model into account and 2) van derWaals (vdW) attraction term. Several procedures have been proposed to evaluate two temperaturedependent parameters $a(T)$ and $b(T)$ appeared in Eq. (15-16) [20-23].

We have determined these parameters from the corresponding states correlation. The temperature-dependent parameters of above equation can be expressed in terms of the following universal functions:

$$
\begin{aligned}
& a(T)=\frac{2 \pi}{3 \rho_{\mathrm{C}}} k T_{\mathrm{C}} F_{\mathrm{a}}\left(T_{r}\right) \\
& a(T)=\frac{2 \pi}{3 \rho_{\mathrm{C}}} k T_{\mathrm{C}} F_{\mathrm{a}}\left(T_{r}\right)
\end{aligned}
$$

Here $\rho_{c}$ is the critical density, and $T_{r}$ is the reduced temperature which is scaled by the critical temperature $T_{C}$. We have presented an empirical formula for universal functions, $F_{a}\left(T_{r}\right)$ and $F_{b}\left(T_{r}\right)$ in terms of reduced temperature, which can be written as:

$$
\begin{gathered}
F_{\mathrm{a}}\left(T_{r}\right)=a_{1} \exp \left(-a_{2} T_{r}\right)+a_{3} \exp \left[-a_{4}\left(T_{r}\right)^{3 / 2}\right] \\
F_{\mathrm{b}}\left(T_{r}\right)=b_{1} \exp \left(-b_{2} T_{r}\right)+b_{3} \exp \left[-b_{4}\left(T_{r}\right)^{3 / 2}\right]
\end{gathered}
$$

where; 
$a_{1}=-0.52305, b_{1}=2.3984$

$a_{2}=-10.964, b_{2}=9.5380$

$a_{3}=3.9201, b_{3}=0.24999$

$a_{4}=-9.9757, b_{4}=-1.1922$.

The mixture version of PHS EOS has the following structure

$$
\frac{P}{\rho k T}=\frac{1+\eta+\eta^{2}-\eta^{3}}{(1-\eta)^{3}}-\frac{\rho}{k T} \sum_{i}^{m} \sum_{j}^{m} x_{i} x_{j} a(T)_{i j}
$$

Where $x_{i}$ and $x_{j}$ are the mole fractions of $i$ 'th and $j$ 'th components, respectively. $\eta$ is the packing fraction of mixtures of hard sphere. This parameter is defined by the following expression

$$
\eta=\frac{\rho}{4} \sum_{i}^{m} x_{i} b(T)_{i}
$$

In the case of binary mixtures, the hard-sphere co-volumes, $b(T)_{i j}$ are additive according to the following expression

$$
b(T)_{i j}=1 / 8\left[b(T)_{i}^{1 / 3}+b(T)_{j}^{1 / 3}\right]
$$

The attractive forces between two hard-sphere species of mixture including $\mathrm{i}$ and $\mathrm{j}$ components can be written as follows

$$
a(T)=\frac{2 \pi}{3\left(\rho_{C}\right)_{j}} k\left(T_{C}\right)_{y}\left(F_{a}\right)_{i j}
$$

In this study, we have applied the following combining rules for $T_{C}, \rho_{C}$ and universalized function $F_{a}$ :

$$
\begin{gathered}
T_{\mathrm{Cij}}=\left(T_{\mathrm{C} i} T_{\mathrm{Cj}}\right)^{1 / 2} \\
\left(\rho_{C i j}\right)^{-1 / 3}=\frac{1}{2}\left[\left(\rho_{C i i}\right)^{-1 / 3}+\left(\rho_{C j j}\right)^{-1 / 3}\right] \\
\left(F_{a}\right)_{i j}=\left[\left(F_{a}\right)_{i i}\left(F_{a}\right)_{j j}\right]^{1 / 2}
\end{gathered}
$$

In this paper in order to examine the effect of different mixing rules on the predicted values of thermodynamic properties, we chose some of our previously studied of binary mixtures consist of:

1) $\mathrm{Ar}-\mathrm{Kr}$ as a noble gas mixture,

2) Mixtures of Xenon+Ethene and Xenon+ Propane,

3) Refrigerant mixtures of R32+R143a as representatives of slightly polar mixtures.

4) Mixtures of $\mathrm{THF}+[\mathrm{BMIM}]\left[\mathrm{PF}_{6}\right]$, $\mathrm{DMSO}+[\mathrm{BMIM}]\left[\mathrm{PF}_{6}\right], \quad[$ Emim $][$ Triflate $]+$ Propanol,
[Emim][Triflate]+Water as representatives of polar mixtures.

It is obvious that in order to calculate the binary potential parameters for each mixture, we need to know the scaling parameters, $\sigma$ and $\varepsilon$ for pure components.

The main objective of this work is to assess the effect of these five mixing rules on the results in our previous works

We took pure component parameters $\sigma$ and $\varepsilon$ for we calculated the values of $\sigma$ from the following equation

$$
=0.809\left(\mathrm{~V}_{\mathrm{c}}\right)^{1 / 3}
$$

Where $\mathrm{Vc}$ is the critical volume. The values of $\varepsilon$ were evaluated using

$$
\frac{\varepsilon}{K}=\frac{T c}{1.2593}
$$

\subsection{Improvement of Our Results}

Also in the last section of this research we tested the effect of two interaction parameters, $\mathrm{K}_{\mathrm{ij}}$ in the results. Two interaction parameters are often needed for complex polar systems. We selected some mixtures of our previous works and repeated our calculation for density and vapor pressure with $\mathrm{K}_{\mathrm{ij}}$ and then compare the new results with previous results. The results are listed in Tables 4-8.

In Table 1 we have shown the calculated values of collision diameters and well depths of pure compound via five different mixing rules.

Table 1. Collision Diameters and Well Depths for the Pure Component, Comprising Our Studied Mixtures.

\begin{tabular}{lll}
\hline Pure component & $\boldsymbol{\sigma} / \mathbf{A}$ & $\mathbf{( \varepsilon / \mathbf { k } _ { \mathbf { B } } ) / \mathbf { K }}$ \\
\hline $\mathrm{Ar}$ & 3.35 & 141.5 \\
$\mathrm{Kr}$ & 3.59 & 140.2 \\
$\mathrm{R} 32$ & 4.02 & 314.878 \\
$\mathrm{R} 143 \mathrm{a}$ & 4.691 & 352.87 \\
$\mathrm{Xe}$ & 3.82 & 142.3 \\
Ethane & 4.371 & 241.9 \\
Propane & 4.721 & 353.35 \\
[BMIM][PF 6$]$ & 0.9669 & 926.28 \\
[Emim][Triflate] & 0.92634 & 808.33 \\
\hline
\end{tabular}

Table 2 contain the AAD of densities for some mixtures

\begin{tabular}{|c|c|c|c|c|c|}
\hline Mixtures & Lorentz-Berthelot & Halgren HHG & Tang-Toennies & Waldman-Hagler & Fit (MADAR) \\
\hline $\mathrm{He}+\mathrm{Xe}$ & 0.5 & 0.499 & 0.502 & 0.497 & 0.462 \\
\hline $\mathrm{R} 32+\mathrm{R} 143 \mathrm{a}$ & 0.2 & 0.201 & 0.199 & 0.189 & 0.162 \\
\hline $\mathrm{THF}+[\mathrm{BMIM}]\left[\mathrm{PF}_{6}\right]$ & 1.05 & 1.01 & 1.002 & 0.995 & 0.862 \\
\hline $\mathrm{DMSO}+\left[\mathrm{BMIM}^{-}\left[\mathrm{PF}_{6}\right]\right.$ & 0.46 & 0.482 & 0.452 & 0.449 & 0.365 \\
\hline$[$ Emim] $]$ Triflate]+Propanol & 0.25 & 0.251 & 0.243 & 0.241 & 0.226 \\
\hline$[$ Emim] $]$ Triflate]+Water & 0.46 & 0.451 & 0.442 & 0.421 & 0.385 \\
\hline
\end{tabular}
such as $\left(\mathrm{He}+\mathrm{Xe}-\mathrm{R} 32+\mathrm{R} 143 \mathrm{a}-\mathrm{THF}+[\mathrm{BMIM}]\left[\mathrm{PF}_{6}\right]-\right.$ $\mathrm{DMSO}+[\mathrm{BMIM}]\left[\mathrm{PF}_{6}\right]-[$ Emim $][$ Triflate $]+$ Propanol-

[Emim][Triflate]+Water) by using different mixing rules. IN this grope we have Noble gases and refrigerant and ionic liquids Mixtures of ionic liquid are with polar solutions. We compere different mixing rules and observed the Fit (MADAR) give better results.

Table 2. The AAD of densities for some mixtures by using different mixing rules. 
Table 3 show the AAD of excess molar volume for the mixtures in this case also Fit (MADAR) give more acceptable results. And Table 4 indicated the AAD of vapor pressure for some above mixtures and the same results repeat.

Table 3. The AAD of excess molar volume for some mixtures by using different mixing rules.

\begin{tabular}{|c|c|c|c|c|c|}
\hline Mixtures & Lorentz-Berthelot & Halgren HHG & Tang-Toennie & Waldman-Hagler & Fit (MADAR) \\
\hline Xenon+Ethene & 0.2 & 0.203 & 0.197 & 0.192 & 0.175 \\
\hline $\mathrm{THF}+[\mathrm{BMIM}]\left[\mathrm{PF}_{6}\right]$ & 0.62 & 0.612 & 0.602 & 0.589 & 0.51 \\
\hline $\mathrm{DMSO}+[\mathrm{BMIM}]\left[\mathrm{PF}_{6}\right]$ & 0.98 & 0.971 & 0.953 & 0.942 & 0.751 \\
\hline$[$ Emim $][$ Triflate $]+$ Propanol & 0.95 & 0.936 & 0.925 & 0.903 & 0.824 \\
\hline$[$ Emim $][$ Triflate $]+$ Water & 1.16 & 1.012 & 0.992 & 0.956 & 0.865 \\
\hline
\end{tabular}

A close look at the results reveals that all mixing rules give almost the same AAD, when the two mixture components are similar in size.

In continues we used two interaction parameters, $\mathrm{K}_{\mathrm{ij}}$ and observed that the results became better and $\mathrm{AAD}$ decreased.
In this part we use 40 different mixtures and compere our results with previous works and we became confident that if we use $K_{i j}$ the accuracy of our results is more than before. The results are listed in Tables 4-8.

Table 4. The AAD of vapor pressure for some mixtures by using different mixing rules.

\begin{tabular}{lllll}
\hline Mixtures & Lorentz-Berthelot & Halgren HHG & Tang-Toennie & Waldman-Hagler \\
\hline Xenon+Ethene & $2 \pm$ & 2.021 & 1.985 & 1.9231 \\
Xenon+Propan & $2.1 \pm$ & 2.004 & 1.9923 & 1.658 \\
\hline
\end{tabular}

Table 5. AAD of the calculated densities $P\left(\mathrm{~kg} / \mathrm{m}^{3}\right)$ of several mixtures of ILs using two interaction parameter.

\begin{tabular}{|c|c|c|c|}
\hline Mixtures & $\mathbf{A A D}(\rho)$ & $\mathbf{K}_{\mathrm{ij}}$ & AAD (new) \\
\hline$[$ EMIM] $]\left[\right.$ Etso $\left._{4}\right]+$ aceton & 0.37 & -0.095 & 0.33 \\
\hline$\left[\right.$ EMIM] $\left[\right.$ Etso $\left._{4}\right]+$ acetonitrile & 0.39 & -0.078 & 0.34 \\
\hline$\left[\right.$ EMIM] $\left[\mathrm{Etso}_{4}\right]+$ dihydromethne & 0.27 & -0.069 & 0.21 \\
\hline$[\mathrm{EMIM}]\left[\mathrm{Etso}_{4}\right]+$ ethenol & 0.24 & -0.046 & 0.19 \\
\hline$\left[\right.$ EMIM] $\left[\right.$ Etso $\left._{4}\right]+$ methanol & 0.28 & -0.065 & 0.21 \\
\hline$\left[\right.$ EMIM] $\left[\right.$ Etso $\left._{4}\right]+$ propylen carbonate & 0.15 & -0.236 & 0.11 \\
\hline$[$ EMIM $]\left[\mathrm{Etso}_{4}\right]+$ water & 0.27 & -0.362 & 0.22 \\
\hline Ethanol (1) $+[\mathrm{Omim}]\left[\mathrm{BF}_{4}\right](2)$ & 0.3 & 0.0047 & 0.24 \\
\hline$\left[\mathrm{C}_{4} \mathrm{MIM}\right]\left[\mathrm{BF}_{4}\right]+$ Water & 0.32 & -0.403 & 0.29 \\
\hline$\left[\mathrm{C}_{4} \mathrm{MIM}\right]\left[\mathrm{BF}_{4}\right]+$ ethanol & 0.27 & -0.043 & 0.22 \\
\hline$\left[\mathrm{C}_{4} \mathrm{MIM}\right]\left[\mathrm{C}(\mathrm{CN})_{3}\right]+$ Water & 0.48 & -0.466 & 0.39 \\
\hline$\left[\mathrm{C}_{4} \mathrm{MIM}\right]\left[\mathrm{C}(\mathrm{CN})_{2}\right]+$ Water & 0.2 & -0.441 & 0.18 \\
\hline$\left[\mathrm{C}_{2} \mathrm{MIM}\right][$ Triflate $]+$ methnol & 0.19 & -0.079 & 0.15 \\
\hline$\left[\mathrm{C}_{2} \mathrm{MIM}\right][$ Triflate $\left.]\right]+$ ethanol & 0.21 & 0 & 0 \\
\hline$\left[\mathrm{C}_{2} \mathrm{MIM}\right][$ Triflate $\left.]\right]+$ ethanol & 0.61 & -0.366 & 0.54 \\
\hline$\left[\mathrm{C}_{2} \mathrm{MIM}\right][$ Triflate $]+1$-Propanol & 0.42 & 0.0279 & 0.36 \\
\hline$\left[\mathrm{C}_{4} \mathrm{MIM}\right]\left[\mathrm{PF}_{6}\right]+\left[\mathrm{C}_{4} \mathrm{MIM}\right]\left[\mathrm{BF}_{4}\right]$ & 0.33 & 0.345 & 0.27 \\
\hline$\left[\mathrm{C}_{4} \mathrm{MIM}\right]\left[\mathrm{BF}_{4}\right]+\left[\mathrm{C}_{4} \mathrm{MIM}\right]\left[\mathrm{MeSO}_{4}\right]$ & & 0.412 & \\
\hline
\end{tabular}

Table 6. Calculated densities of several mixtures of alkanes using two interaction parameter.

\begin{tabular}{llll}
\hline Mixtures & $\boldsymbol{\rho}(\mathbf{k g} / \mathbf{m} 3)$ & $\boldsymbol{\rho}(\mathbf{n e w})$ & $\mathbf{K}_{\mathbf{i j}}$ \\
\hline Toluene+Pentan & 0.6598 & 0.6143 & 0.1236 \\
Toluene+Hexane & 0.7445 & 0.7231 & 0.1298 \\
Toluene+Heptan & 0.7504 & 0.7136 & 0.2103 \\
Toluene+Octane & 0.7687 & 0.7214 & 0.2563 \\
Toluene+Nonane & 0.7112 & 0.6932 & 0.3695 \\
Toluene+Decane & 0.7464 & 0.7325 & 0.3789 \\
\hline
\end{tabular}

Table 7. Calculated densities of several mixtures of nobel gases and refrigerants using two interaction parameter.

\begin{tabular}{llll}
\hline Mixtures & $\boldsymbol{\rho}\left(\mathbf{k g} / \mathbf{m}^{\mathbf{3}}\right)$ & $\boldsymbol{\rho}(\mathbf{n e w})$ & $\mathbf{K}_{\mathbf{i j}}$ \\
\hline propan- $\mathrm{C}_{32}$ & 0.62 & 0.60 & 0.32 \\
$\mathrm{He}+\mathrm{Xe}$ & -1.19 & -1.02 & 0.14 \\
$\mathrm{R}_{32}+\mathrm{R}_{143 \mathrm{a}}$ & -1.87 & -1.23 & 0.37 \\
$\mathrm{R}_{134 \mathrm{a}}+\mathrm{R}_{290}$ & -1.17 & -1.01 & 0.41 \\
$\mathrm{R}_{32}+\mathrm{R}_{125}$ & -1.70 & -1.33 & 0.23 \\
\hline
\end{tabular}

Table 8. Calculated densities and vapor pressure of several mixtures of Nobel gases with alkanes, and ILs with alcohols. Using two interaction parameter.

\begin{tabular}{llll}
\hline Mixtures & P/Mpa & P/Mpa (new) & Kij \\
\hline $\mathrm{CO} 2+\mathrm{R} 134$ & 0.87 & 0.83 & 0.324 \\
Xe+Ethane & 0.65 & 0.62 & 0.124 \\
Xe+Propane & -1.38 & -1.06 & 0.134 \\
Mixtures & density & with $\mathrm{k}_{\mathrm{ij}}$ & $\mathrm{K}_{\mathrm{ij}}$ \\
benzene +[EMIM] ${ }^{+}\left[\left(\mathrm{CF}_{3} \mathrm{SO}_{2}\right)_{2} \mathrm{~N}\right]^{-}$ & 0.95 & 0.91 & 0.0241 \\
$\mathrm{Cyclohexane}^{-}[\mathrm{EMIM}]^{+}$ & 0.74 & 0.72 & 0.0348 \\
{$\left[\left(\mathrm{CF}_{3} \mathrm{SO}_{2}\right)_{2} \mathrm{~N}\right]^{-}$} & & & \\
$\mathrm{C}_{6} \mathrm{H}_{6}+\mathrm{C}_{12} \mathrm{H}_{30}$ & 0.91 & 0.80 & 0.0233 \\
$\mathrm{CH}_{3} \mathrm{OH}+[\mathrm{BMIM}]\left[\mathrm{Ntf}_{2}\right]$ & -1.54 & -1.21 & -0.006 \\
$\mathrm{C}_{2} \mathrm{H}_{5} \mathrm{OH}+[\mathrm{BMIM}]\left[\mathrm{Ntf}_{2}\right]$ & -1.43 & -1.11 & -0.123 \\
$\mathrm{C}_{3} \mathrm{H}_{7} \mathrm{OH}+[\mathrm{BMIM}]\left[\mathrm{Ntf}_{2}\right]$ & -1.54 & -1.13 & -0.265 \\
$\mathrm{C}_{6} \mathrm{H}_{6}+[\mathrm{BMIM}][\mathrm{Ntf}$ & -1.62 & -1.33 & 0.003 \\
\hline
\end{tabular}

\section{Conclusion}

This study shows that all the mixing rules are acceptable and the mixture properties are affected slightly using different mixing rules, especially when the mixture components do not differ much in size. However, the results showed that Fit (MADAR-2) mixing rule give better results. When the mixture components have considerable difference in their collision diameter and well depth values, the Lorentz-Berthelot mixing rule performs better than other rules. Other rules do not show an obvious trend so that it is not possible to classify them. Also observed using two interaction parameters, $\mathrm{K}_{\mathrm{ij}}$ improve the results and the AAD decreased.

\section{References}

[1] U. Deiters, Fluid Phase Equlibria, 33, 267, 1987.

[2] J. Serb. Chem. Soc. 66 (4), 2001, 213-236. 
[3] Y. S. Wei, R. J. Sadus, AIChE J. 46, 169, 2000.

[4] A. K. Al-Matar, D. A. Rockstraw, J. Comput. Chem. 25, 660, 2004.

[5] M. P. Allen, D. J. Tildesley, Computer Simulation of Liquids, 2nd ed., Oxford University Press, New York, 1989.

[6] W. F. Van Gunsteren, P. K. Weiner, A. J. Wilkinson, Kluwer Academic Publishers, Dordrecht, 1997.

[7] T. A. Halgren, J. Am. Chem. Soc. 114, 7827, 1992.

[8] K. T. Tang, J. P. Toennies, Z. Phys. D: At., Mol. Clusters 1, 91, 1986.

[9] A. K. Al-Matar, Ph. D. Thesis, New Mexico State University, Las Cruces, New Mexico, 2002.

[10] J. Bzowski, J. Kestin, E. A. Mason, F. J. Uribe, J. Phys. Chem. Ref. Data 19, 1179, 1990.

[11] J. Kestin, K. Knierim, E. A. Mason, B. Najafi, S. T. Ro, W. A. Wakeham, J. Phys. Chem. Ref. Data, 13, 229, 1984.

[12] T. H. Chung, M. Ajlan, L. L. Lee, K. E. Starling, Ind. Eng. Chem. Res. 1988, 27, 671.

[13] D. Mohammad-Aghaie, M. M. Papari, J. Moghadasi, and B. Haghighi, Bull. Chem. Soc. Jpn. 2008, 81,. 10, 1219.
[14] Richard Anthony McFarlane, University of Alberta, Fall 2007.

[15] WU. Yugong, Z. XuanheE, F. Zhigang, Journal of Electroceramics, 2003, 11, 227-239.

[16] M. M. Papari, S. M. Hosseini, F. Fadaei-Nobandegani, and J. Moghadasi, Korean J. Chem. Eng, 2012.

[17] G. C. Maitland, M. Rigby, E. G. Smith, W. A. Wakeham, Intermolecular Forces: Their Origin and Determination, Clarendon Press, Oxford, U.K., 1981.

[18] K. T. Tang, J. P. Toennies, J. Phys. Chem. B, 102, 7470, 1998.

[19] S. M. Hosseini, Ionics 16, 571-575, 2010.

[20] Y. Song, E. A. Mason, J. Chem. Phys. 91, 7840-7853, 1989.

[21] Y. Song, E. A. Mason, Fluid Phase Equilib. 75 (1992) 105115.

[22] G. Ihm, Y. Song, E. A. Mason, J. Chem. Phys. 94, 3839-3848, 1991.

[23] L. Maftoon-Azad, H. Eslami, A. Boushehri, Fluid Phase Equilib. 263, 1-5, 2008. 